\title{
Verbal Paired Associates II Subtest (WMS-
} IV)

National Cancer Institute

\section{Source}

National Cancer Institute. Verbal Paired Associates II Subtest (WMS-IV). NCI Thesaurus.

Code $C 120345$

A subtest of the Wechsler Memory Scale, 4th Edition that evaluates verbal memory for associated word pairs. 10 or 14 word pairs are read to the subject. After a delay, the first word of each pair is read, and the subject is asked to provide the corresponding word. 